\title{
Using Pervasive Displays to Aid Student Recall - Reflections on a Campus-Wide Trial
}

\author{
Mateusz Mikusz \\ Lancaster University \\ Lancaster, UK \\ m.mikusz@lancaster.ac.uk
}

\author{
Sarah Clinch \\ University of Manchester \\ Manchester, UK \\ sarah.clinch@manchester.ac.uk
}

\author{
Peter Shaw \\ Lancaster University \\ Lancaster, UK \\ p.shaw@lancaster.ac.uk
}

\author{
Nigel Davies \\ Lancaster University \\ Lancaster, UK \\ nigel@comp.lancs.ac.uk
}

\author{
Petteri Nurmi \\ Lancaster University \\ Lancaster, UK \\ p.nurmi@lancaster.ac.uk
}

\begin{abstract}
University campuses are rapidly transitioning to environments that are rich in technology designed to support learning throughout the day and in diverse forms. Traditional lectures and seminars are supplemented with rich WiFi coverage, integrated learning environments, video lectures, public display networks and other innovations. Of these diverse technologies, the role and potential of public display networks in higher education is currently least understood. Indeed, most campus displays are merely used as means of information dissemination or as tools to support collaboration.

In this paper, we explore the potential of pervasive display technologies as active contributors to university teaching and learning. We describe a real-world explorative study in which campus displays we used to stimulate student recall of lecture material. Our experiences demonstrate that public displays can be leveraged to provide prompts at opportune moments and invites further research in designing memory prompts to leverage the learning potential of pervasive displays in campus settings. The insights we garnered form a solid foundation and highlight opportunities and challenges in the field.
\end{abstract}

\section{ACM Classification Keywords}

H.5.m. Information Interfaces and Presentation (e.g. HCI): Miscellaneous

\section{Author Keywords}

pervasive displays, public displays, deployments, memory augmentation, e-learning

\footnotetext{
Permission to make digital or hard copies of part or all of this work for personal or classroom use is granted without fee provided that copies are not made or distributed for profit or commercial advantage and that copies bear this notice and the full citation on the first page. Copyrights for third-party components of this work must be honored. For all other uses, contact the owner/author(s).

PerDis '18 June 6-8, 2018, Munich, Germany

(C) 2018 Copyright held by the owner/author(s).

ACM ISBN 978-1-4503-5765-4/18/06.

DOI: https : //doi .org/10.1145/3205873. 3205882
}

\section{INTRODUCTION}

University campuses are increasingly embracing information technology as means to foster innovation, and to support and enhance learning [2]. Through this development, campuses are transforming from mere places of education into holistic spaces for learning and innovation. Traditional lectures and seminars are supplemented with virtual learning environments, video recording and podcasting, remote participation, clickers and automated attendance monitoring, whilst physical buildings and campuses are enhanced with a wide-range of devices and services including campus-wide WiFi coverage, IoT and pervasive displays. However, to what extent these technologies actually support and enhance learning is still poorly understood [14]. This is particularly true for pervasive displays - whilst campus deployments of such displays are not uncommon $[11,15,21]$, there is little empirical evidence on the role and usefulness of pervasive displays to enhance and improve learning.

Pervasive displays have a long history as broadcast devices for advertising and other media content. More recently, researchers have begun to explore their potential in impacting cognition $[1,6]$. Particularly relevant is the proposal that such displays could have a key role in memory augmentation [6] - indeed, in their first visions the use of displays as part of a broader memory augmentation architecture, the authors suggest a range of application areas including behaviour change, advertising and learning.

Memory augmentation technologies typically collect and represent cues with the intention of triggering rehearsal and recollection of past or planned events [12, 20]. A variety of studies has shown the potential of such cues in highly constrained learning tasks - e.g. in studies of word associations [22] and flashcard study [8]. However, findings from these studies do not directly translate to campus deployments of pervasive displays: limited attention has been given to peripheral cue presentation [8,9], and the deployments under consideration have typically been single user with carefully controlled cue delivery. Pervasive displays challenge these models with multiple (potentially simultaneous [19]) users 
engaging in peripheral glances of very limited duration [5, 13], and with unpredictable delays between exposure to learning material and opportunities for cue delivery.

In this paper, we contribute to this new research space by exploring the potential of pervasive displays as a memory augmentation tool for enhancing learning in campus environments. We base our considerations on our experiences of conducting a 10 -week, real-world, explorative study that leveraged an established display deployment as a means of presenting memory cues associated with a set of first year undergraduate lectures with the intention of stimulating student recall of lecture material. We specifically target two questions that are fundamental to the successful use of memory cues. Firstly, we examine whether pervasive displays have the potential to reach students at opportune moments by analysing mobility patterns collected through Bluetooth sightings. Results from our analysis show that pervasive displays indeed have potential to deliver cues at relevant moments, but that scheduling needs to be carefully designed to accommodate different contexts and mobility patterns. Secondly, we examine the design of memory cues to maximise their relevance. We compare relevance assessments of teaching material by students and teachers to see to what extent cue delivery would need to be personalised - and to what extent it could simply be determined by teaching staff as part of their preparation of learning materials. Besides examining these two questions, we report the technology used in our trial environment and reflect on experiences from our trial. The insights we garnered form a solid foundation and highlight opportunities and challenges in the field.

\section{THE LANCASTER E-LEARNING MEMORY TRIAL}

We conducted a 10-week trial deployment to develop an understanding of the potential of pervasive displays in supporting and enhancing learning. In this section, we summarise the context of the trial including the campus setting and displays, the technical mechanism used for delivering cues to students, and the experimental protocol followed in the trial.

\section{Trial Environment}

The trial was conducted at Lancaster University, a campus university in North West England. The university has approximately 13,115 students $(9,500$ undergraduates, and 3,615 postgraduates). Many live on campus, whilst others commute in for periods of the day - walking between a variety of university buildings (lecture theatres, cafes, library etc.).

One of the unique characteristics of the university campus is the presence of e-Campus [4], the world's largest and longestserving public display research testbed. This deployment encompasses over 65 large displays (typically around 40-50 inches) in a variety of indoor and outdoor locations across the campus including departmental buildings, student accommodation, lecture theatres and campus walkways (Figure 1). As well as serving as a research platform, the displays are in constant use as a key university signage infrastructure, showing a mix of static content such as college, department and university-wide news stories.

In addition to the e-Campus displays, the university provides a portfolio of technologies to support teaching and learning.
Lectures are video recorded and archived for students to consume asynchronously, and web-based virtual learning environments (VLEs) that provide access to a wealth of services including hosting of complementary lecture materials and interactive formative and summative assessment (e.g. online quizzes). Furthermore, a cross-platform mobile phone application (iLancaster) provides a gateway to large number of university services. Students can access both lecture timetables and materials through iLancaster, and the application also provides automated attendance monitoring that determines if the student is present for compulsory lectures or labs through the phone's detection of Bluetooth Low Energy (BLE) beacons deployed across campus teaching spaces

\section{Platform for Memory Cue Delivery}

To explore the potential application of pervasive displays to aid recall of learning materials, we developed a prototype cue delivery platform to support a longitudinal experimental trial. The platform needed to be capable of delivering lecturerelated material to students registered to specific courses. The developed prototype combined iLancaster, the used VLEs, the e-Campus display network, and a significantly simplified version of the Tacita display personalisation framework [7].

In order to support dynamic delivery of cues to passing students participating in the study, we extended and integrated the e-Campus, iLancaster and Tacita systems, ensuring that we would be able to detect study participants in the vicinity of the displays. Firstly, we extended the physical infrastructure, equipping all deployed screens with BLE beacons that constantly transmit the unique identifier of the co-located display. Secondly, we built upon the existing capability of iLancaster to detect BLE beacons, developing a location service, distinct from the automated attendance monitoring, that would detect beacon transmissions even whilst a phone was on standby. Upon detecting a beacon, the iLancaster location service would immediately send a request to the Tacita backend component including the unique identifier that was received as part of the beacon transmission. Finally, upon receipt of the request, the e-Campus backend then triggered an update in the content shown on the nearby display, leading the lecture-related material to be shown to students for as long as Tacita continued to report proximity.

\section{Trial Design}

A 5-week pilot informed the design of our trial. Following the pilot, we conducted a 10 week within-subjects study containing two experimental blocks separated by a control block. During each experimental block, taught lectures were followed by an eight-day cue delivery period in which Tacita was used to show cues to participating students; during periods where no participating students were identified as being proximate the screens continued to show the regular schedule of content. During control blocks, no cues were delivered to displays the screens showed only the regular schedule of content.

Students were recruited from a cohort of $\sim 180$ first year computer science undergraduates attending a weekly one-hour introductory course on programming (no additional demographics were collected), and invited to activate the Tacita 


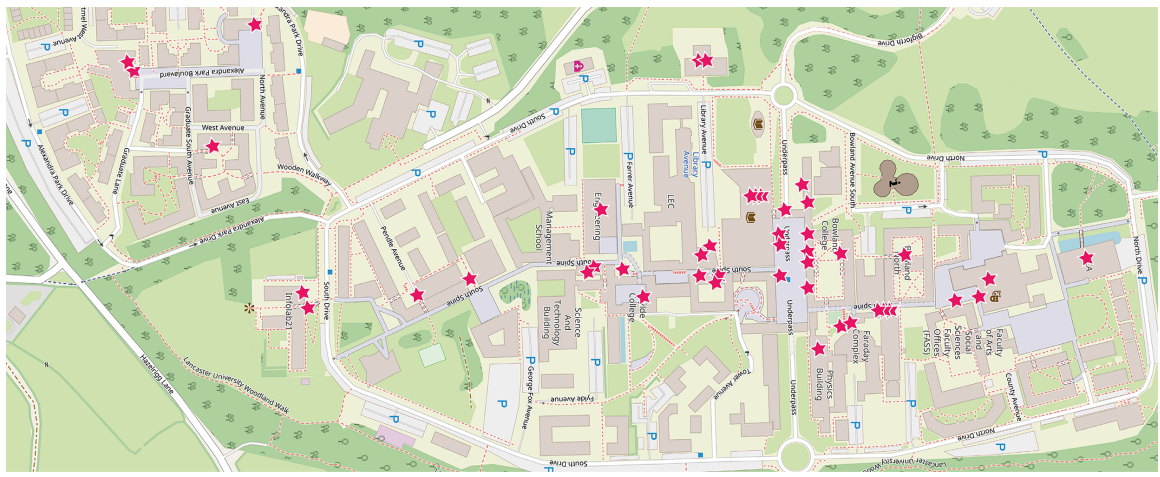

Figure 1. Overview of deployed public displays at the Lancaster University campus.

location service integration into iLancaster. Participating students incentivised with entry into a prize draw - one entry for use of the application, plus one further entry per formative assessment completed. The study was subject to institutional and project ethical approval processes, and all students were required to provide informed consent prior to participation.

At weekly intervals, students were prompted to complete a formative assessment on the previous week's lecture content (content presented 9-10 days previously), and a short questionnaire about the actions they had taken to revise the material that week (including whether they had attended the lecture, and whether they were aware of having seen related content on the screens). The formative assessment consisted of eight short free-text questions that were scored from $0-100 \%$ by a single assessor. To enable analysis of the impact of the memory augmentation system during the experimental blocks, four of the questions covered content that was delivered through memory cues to students, while the remaining four questions covered content that was taught in the associated lecture but not delivered out through the memory augmentation system. These assessments were conducted as part of the students' additional weekly practical classes that covered in-depth content of the previous week's lecture.

\section{Cue Selection}

Having developed a technical means for delivering cues to students through proximate pervasive displays, we then needed a mechanism for developing salient cues that could be presented on this platform. Although recall quality is evidently influenced by the properties of the cues used, there was little in the literature that could help inform this design process. With no theory or prior experience reports to draw on, we opted for a co-design approach in which we asked ten students in each lecture to note down the four most important lecture slides and then used these as input for the development of a set of four static images.

We recruited ten students prior to the commencement of each taught lecture. Each student was asked to identify four lecture slides that they considered to be the most important and useful for summarising the topic covered during that hourlong session. The resulting answers were pooled to develop a ranking of the most-valued slides. The top four slides were then selected and used these as a basis for the creation of a set of visual memory cues. Four such cues were created, each based on one of the four rated slides. The cues all followed the same visual layout, that featured a screenshot of the relevant slide inset into a photograph of the slide's delivery within the lecture. An example of one such visual memory cue is shown in Figure 2.

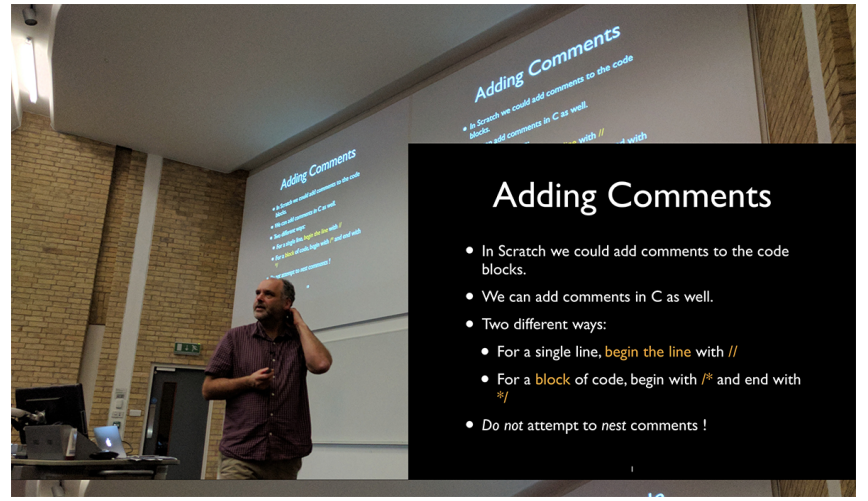

Figure 2. Example memory cue consisting of the context of the lecture and the relevant lecture slide.

\section{EXPERIENCES AND ANALYSIS}

As the first study of its kind, our trial had the potential to offer insights in three distinct areas. Firstly, on the feasibility of pervasive display infrastructure as a cue delivery platform for memory augmentation. Prior research [6] has assumed that displays could be leveraged to deliver the right type of cue at the right moment, but no trial has attempted to confirm if this could be achieved. Secondly, on the development of relevant learning cues, suitable for presentation on a public display. Finally, on the outcome itself - would our initial attempts to cue lecture content through displays have an effect on learning, and how might such effects be measured?

In this section we consider each of these areas in turn, drawing on evidence from our trial. During this process, we note that our study did not produce a noticeable uplift in the learning measured in our formative assessment.

\section{Cue Delivery}

To understand how the mobility of the students influences the potential for delivering cues, we compare the spatial distributions of memory cue delivery requests across times when 


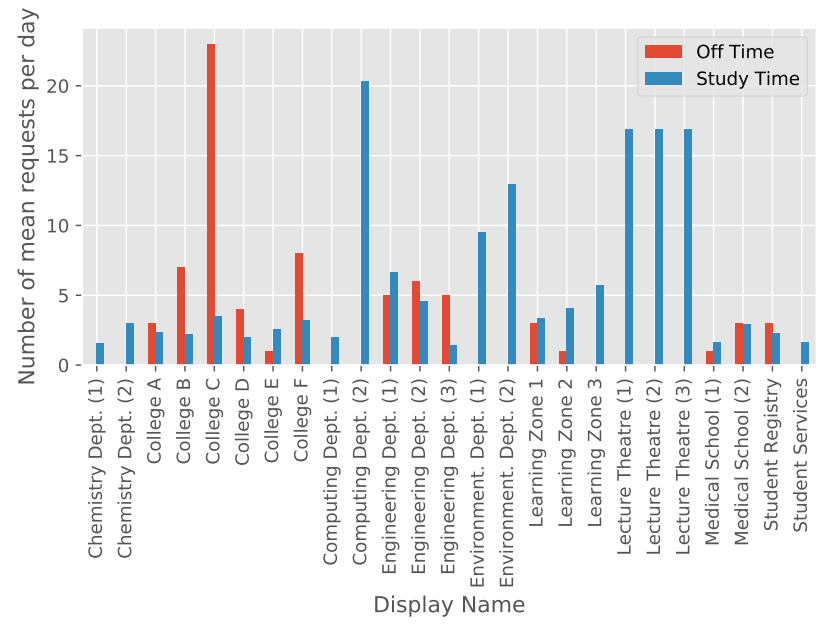

Figure 3. Comparison of locations in which content was requested during potential study time and in their free time.

students typically spend time on campus for learning purposes (i.e. between $9 \mathrm{am}$ and $6 \mathrm{pm}$, Monday to Friday) and times when they are more likely to be on-campus for social and recreational reasons (during evenings and weekends).

Figure 3 compares the spatial distribution of content requests between these study and leisure periods, whilst Figure 4 shows differences between weekdays and weekends. Both figures show mobility patterns to be largely predictable. Indeed, during study periods, we observe the majority of content requests at displays near lecture theatres, study areas and the library, while in off-study times the displays around student accommodation and social areas (colleges) dominate. We observe a similar trend between weekdays and weekends, with weekdays dominated by locations visited during study time, and weekends featuring spaces more likely to be visited in recreational time. Generally, we observe noticeably fewer requests during the weekends - suggesting fewer or less-mobile students during weekends, with the resulting reduced exposure to memory cues as compared to weekdays.

From this analysis we make two important observations. Firstly, both the spatial and temporal distributions of the requests suggest that cue delivery schedules can be designed to maximise the likelihood of being exposed to the cue and to minimise time to "first-exposure", i.e. to the first sighting of the memory cue after the lecture. This is particularly important for reinforcing knowledge retention. Secondly, the results correlate well with expected activity patterns of students, suggesting that whilst personalised systems such as Tacita may be beneficial, there is still substantial potential for delivering display-based learning cues on campuses without this technology. Furthermore, cues could be tailored for the expected locations, showing content for extended periods within accommodation and leisure spaces to maximise the potential of user glancing and paying attention to the content, while displays located close to lecture areas could be used to minimise the time to first exposure and be reminded of the lecture context.

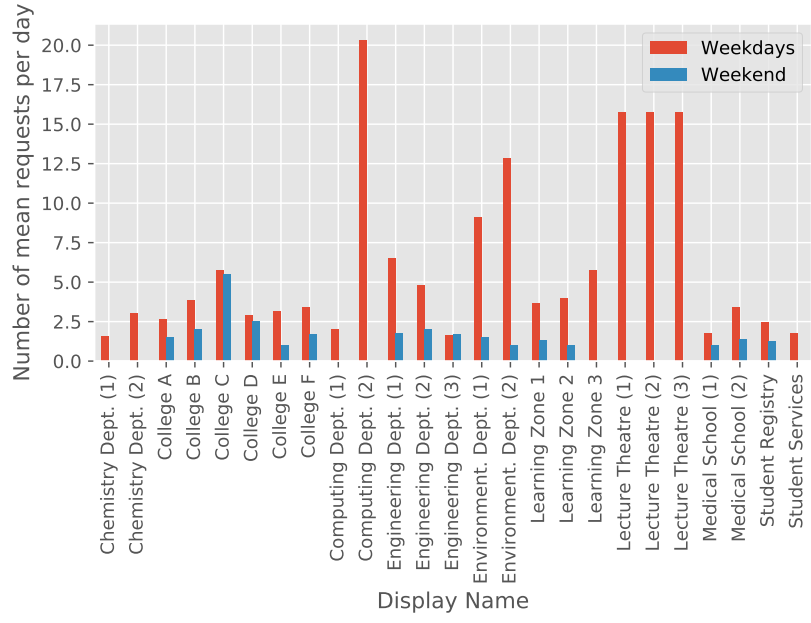

Figure 4. Comparison of content requests and locations on weekends and weekdays.

\section{Capture and Content Creation}

The creation of relevant and appropriate content for the use as memory cues is a fundamental first step for memory augmentation. To determine appropriate content to serve as memory cues, we asked students in each lecture to note down the four slides that will best help you remember the overall content of the entire lecture and created a scoring of slides based on the participants' selections. We collected a total of 337 scores over the ten week study period from a changing set of ten participants each week that resulted in 40 "most important" lecture slides (4 per lecture). These scores enabled us to perform a qualitative analysis of the kinds of content participants selected as their most important slides, and conduct a comparison against an expert opinion from a member of staff who had taught on the course.

Student ratings showed considerable diversity. Typically, slides rated as most important received only an average of 2.1 scores, i.e. only two out of ten participants selected the same slide $(\mathrm{SD}=1.25)$. Despite this, there were obvious trends in the kinds of content students selected: (1) slides with lists of key points to remember, such as best programming practises or guidelines for writing 'beautiful' code; (2) annotated code snippets with fundamental and relatively complex programming paradigms such as pointers, array manipulations and loops; and (3) explanations and citations. Content that appeared to be significantly less valued by students included slides containing images, single facts, and details regarding coursework and assessments. Students selected content equally across the entire duration of the lecture - we did not observe any tendency for participants to select memorable slides only from the beginning or end of the lecture - the summary slides were among the four highest rated slides in just two of the 10 lectures. Interestingly, the final lecture of the course (a revision lecture) triggered the highest similarities in slide scores $(\mathrm{M}=1.43, \mathrm{SD}=0.66)$.

To conduct a qualitative comparison of the content selected by students with an expert opinion, we asked one of the two members of staff teaching on the course to also provide a 
scoring of four slides for each lecture. The task followed the same format as the student scoring, with the staff member selecting the top four slides that they though would best help students remember the overall content of the entire lecture. Generally, we observed that while both students and experts choose slides of the types (1, slides featuring key points) and (2, annotated code snippets), in only six cases the selection of the 'most important' slide overlapped with the participants' choices (of a total of 40 rated important slides), and in only 12 cases the expert has chosen content that received at least one score by participants (of a total of 166 lecture slides that received at least one score). Whilst the types of memory cues were similar in two of the four content groups, these results show a noticeable discrepancy in the actual content and topics students believe to be both important and appropriate as memory cues compared to the expert's opinion. We further found that the expert tended to focus on content that featured figures and images, and other content that included 'bold' or easy to remember quotes, e.g. "programs that aren't tested are worse than useless" - a type of content that typically received few or no scores from students.

In contrast to the observation in the previous section that personalisation may not be necessary for delivering display-based memory cues, the variance in content ratings across students suggests that maximising the relevance of the cues would require personalising the content that is shown for each individual student. However, our results also highlight a conflict between what is considered educationally relevant, i.e. what is recommended as relevant by teaching staff, and what is considered personally relevant by the students. How to best handle this discrepancy remains an open research issue. Showing personalised content may help to catch the user's attention more easily, whereas showing content that is deemed relevant by teaching staff may have a higher overall impact on learning performance. Another possibility would be to use personalised cues to engage the user's attention, and then switch to a secondary cue that is educationally relevant.

\section{Evaluation}

To assess the impact and success of the memory augmentation system, we analysed the responses to the formative assessment of previous weeks' content, comparing participant performance between control weeks and experimental blocks. As measure of performance, we considered a numerical rating assigned to each of the student's responses. The scores were assigned by a member of the teaching staff and were between 0 and $100 \%$. For the analysis, we first eliminated the set of responses from participants who only conducted a single formative assessment and were therefore only exposed to either the control or experimental blocks (25 students). We used the remaining set of responses to measure the impact on the performance of (1) the participants overall mean performance of all responses from experimental weeks to control weeks, and (2) the participants performance within experimental week comparing the four questions that were covered by the memory augmentation system, and the remaining four questions regarding content taught in the lecture but not delivered by the memory augmentation system.
Our initial results show that students performed nearly identical for both experimental weeks $(\mathrm{M}=0.71, \mathrm{SD}=0.20)$ and control weeks $(\mathrm{M}=0.73, \mathrm{SD}=0.16)$ and did not show a significant impact on the participant's performance. The number of students participating in the experiment decreased across the weeks, which made it impossible to consistently measure the influence of the memory cue pilot and development of student performances over a longer period of time.

Another concern for assessing the memory effect was the difficulty to isolate the effect from the memory augmentation system to additional factors that might have impacted the participant's performance in the assessments. For example, due to the ethical guidelines we had to ensure that both the memory cues and formative assessments were of relevance to the lecture the participant was conducting and had no negative impact on their course performance. Therefore, delivered and assessed content were based on the associated lecture that a participant has visited and potentially also revised prior to conducting the study. In addition, certain participants might perform generally better in the assessment due to their greater or preexisting knowledge of the lecture content compared to other participants. Their knowledge and additional revises are factors that cannot be excluded or isolated in this experiment but have potentially a large impact on our ability to measure the success of the system.

\section{DISCUSSION}

\section{Limitations}

Evaluating cue effectiveness directly is rather challenging. In our case the cues were directly linked to the teaching process, and hence any potential effects would be moderated by differing student performance levels - and level of motivation. Secondly, whilst we were able to capture each occurrence of participants requesting personalised memory cues due to their proximity to a display, and the delivery of the corresponding memory cue through our display network, we were unable to detect whether participants have actually noticed and seen the memory cue on the display when passing by. Capturing display views on displays would have required us to equip each display with a suitable video analytics software and recognise participants on the video - a significant invasion of participants' privacy.

We note that the memory augmentation system was designed as a supplementary system as part of an overall electronic learning environment for students. We believe that showing a significant improvement of the participants' performance is not necessarily required. Instead, we were able to integrate the public display deployment on the university campus as part of the students' learning experience and brining together two initially distinct systems - with a clear benefit for the student.

\section{Memory Cue Delivery}

While the results of our trial highlighted the potential of delivering memory cues to students, it also highlighted how difficult it is to design cues that effective in aiding learning. None of the students acknowledged seeing the cues during those weeks that they were shown, even if the sighting patterns would suggest they had seen them. This is potentially due to the content not being salient enough to attract sufficient attention from the 
students. Indeed, several studies have highlighted how people tend to quickly glance at displays before turning their attention away $[13,18]$, suggesting that designing the cues so that users can quickly identify them during these short glances would be central to the success of cues. Another potential way to improve the effectiveness of the cues would be to design them to be interactive. Interactivity can help draw people around the display [19], and it has also been shown to have a positive impact on memory recall [1]. However, such designs are typically most effective in places where there is dedicated space for interacting with the content, which is generally not the case for campus deployments.

While we explicitly considered the relevance of content shown on the displays, our results also highlighted that relevance is likely to be highly personal even in educational context. Indeed, results of the content ratings highlighted how most students had differing views of what is important. Investigating personalised cues instead of showing the same content for all students would be another avenue worth investigation.

\section{Learning Theories}

Another interesting exploration would be to consider other types of learning theories. For example, stimulated recall [3] uses cues that shown the learning context in a bid to engage the same cognitive processes that were involved during the lecture setting. Some studies of stimulated recall have used autographical content, such as pictures taken by students, as the cue to stimulate later recall [10]. In our context, this approach could be translated into cues that use personal relevance, but also show the classroom context more explicitly.

\section{Memory Augmentation in other Domains}

Whilst the e-Campus platform was used to underpin this paper (as described in the Trial Environment section), there have also been a number of attempts that explored the use of public displays on university campuses and learning environments. For example, Ojala et al. [17] installed multipurpose interactive screens in varied environments within the city of Oulu, Finland, including the municipal service centre and the main library. Whilst the authors used their system to deploy a variety of sensors and services for the audience, the authors used the system to analyse the user behaviour over a three year period [16]. We believe a memory augmentation system that benefits the passers-by as introduced in our work can be used as an additional service for an audience with the ultimate goal to increasing their attention toward and engagement with the display deployment - increasing the overall value of the system. Memarovic et al. [15] investigated the design of "interacting places" that accommodate the current communication ecology of students and conducted a series of interviews. The results show that participants considered public displays as the most appropriate medium for posting and viewing news and events, similar to the kinds of content that could also be found on social media pages of communities. In a further example, Greis et al. [11] investigated viewer expectations of content moderation delays for user generated content. The authors developed and deployed a web-based application on a display located on the university campus that allowed students to tweet images to the display that were shown after a short delay introduced by the moderation process. We believe that both [15] and [11] are examples that express the potential of increasing the value of displays to viewers by delivering more relevant content - our deployment follows in this pattern by having users of the system (students) contribute to content generation to increase relevance.

\section{CONCLUSIONS}

This paper explored the potential of using pervasive displays as active contributors to teaching and learning in university environments, focusing specifically on their role and potential as memory augmentation tools. We used data collected from a 10-week trial deployment to investigate two key questions in the design of memory augments: how mobility shapes the potential of delivering cues, and how to design memory cues that are relevant.

Our analysis of mobility patterns suggest that students movements are largely predictable, and are reflective of their social and spatial context. These patterns have the potential for use in memory cue design and presentation, to tailor information based on contextual data and to ensure cue presentation schedules that maximise the potential of reaching students regularly and at sufficiently regular intervals. In terms of content design, our results highlight a large variance in content ratings across students, and discrepancy between expert and student assessments. These differences suggest a need for further investigation into the design of memory cues that remain relevant, whilst delivering pedagogic value, i.e. ensuring effectiveness in reinforcing learning. Finally, we assessed the effectiveness of memory cues during our trial deployment, highlighting several issues that reduced the impact of cues. These include insufficient granularity of location information, coupling of memory cues with learning performance, and limited attention paid to the presented cues.

We believe our trial offers some indication that pervasive displays have the potential to facilitate and enhance learning in university settings. However, we have also shown that there are several open challenges related to how to best design and deliver cues in order to maximise their impact on learning. Nevertheless, our work provides an important first step in explorations of pervasive displays as memory augmentation tools in educational contexts.

\section{ACKNOWLEDGMENTS}

This research is partially funded through the Future and Emerging Technologies (FET) programme within the 7th Framework Programme for Research of the European Commission, under FET grant number: 612933 (RECALL), and by the UK EPSRC under grant numbers EP/N028228/1 (PACTMAN) and EP/N023234/1 (PETRAS IoT Research Hub - Cybersecurity of the Internet of Things).

\section{REFERENCES}

1. Florian Alt, Stefan Schneegass, Michael Girgis, and Albrecht Schmidt. 2013. Cognitive Effects of Interactive Public Display Applications. In Proceedings of the 2Nd ACM International Symposium on Pervasive Displays (PerDis '13). ACM, New York, NY, USA, 13-18. DOI : http://dx.doi.org/10.1145/2491568.2491572 
2. S. Adams Becker, M. Cummins, A. Davis, A. Freeman, C. Glesinger Hall, and V. Ananthanarayanan. 2017. NMC Horizon Report: 2017 Higher Education Edition. Technical Report. The New Media Consortium, Austin, Texas. https://www. learntechlib.org/p/174879

3. J. Calderhead. 1981. Stimulated Recall: A Method for Research on Teaching. British Journal of Educational Psychology 51, 2 (1981), 211-217. DOI: http://dx. doi.org/10.1111/j.2044-8279.1981.tb02474.x

4. Sarah Clinch, Nigel Davies, Adrian Friday, and Christos Efstratiou. 2011. Reflections on the Long-term Use of an Experimental Digital Signage System. In Proceedings of the 13th International Conference on Ubiquitous Computing (UbiComp '11). ACM, New York, NY, USA, 133-142. DOI :

http://dx.doi .org/10.1145/2030112.2030132

5. Nicholas S. Dalton, Emily Collins, and Paul Marshall. 2015. Display Blindness?: Looking Again at the Visibility of Situated Displays Using Eye-tracking. In Proceedings of the 33rd Annual ACM Conference on Human Factors in Computing Systems (CHI '15). ACM, New York, NY, USA, 3889-3898. DOI :

http://dx.doi.org/10.1145/2702123.2702150

6. Nigel Davies, Adrian Friday, Sarah Clinch, Corina Sas, Marc Langheinrich, Geoff Ward, and Albrecht Schmidt. 2015. Security and privacy implications of pervasive memory augmentation. IEEE Pervasive Computing 14, 1 (2015), 44-53.

7. Nigel Davies, Marc Langheinrich, Sarah Clinch, Adrian Friday, Ivan Elhart, Thomas Kubitza, and Bholanathsingh Surajbali. 2014. Personalisation and Privacy in Future Pervasive Display Networks. In Proceedings of the SIGCHI Conference on Human Factors in Computing Systems (CHI '14). ACM, New York, NY, USA, 2357-2366.

8. Tilman Dingler, Corinna Giebler, Ulf Kunze, Tim Wúrtele, Niels Henze, and Albrecht Schmidt. 2016a. Memory Displays: Investigating the Effects of Learning in the Periphery. In Proceedings of the 5th ACM International Symposium on Pervasive Displays (PerDis '16). ACM, New York, NY, USA, 118-123. DOI : http://dx.doi.org/10.1145/2914920.2915030

9. Tilman Dingler, Philipp Wahju, Pascal Knierim, and Albrecht Schmidt. 2016b. Memory Support through In-Home Display Deployment. IT Professional 18, 6 (2016), 42-49.

10. Wendy Fox-Turnbull. 2009. Stimulated recall using autophotography-A method for investigating technology education. Strengthening the position of technology education in the curriculum. Delft, The Netherlands: International Technology and Engineering Educators Association (2009).

11. Miriam Greis, Florian Alt, Niels Henze, and Nemanja Memarovic. 2014. I Can Wait a Minute: Uncovering the Optimal Delay Time for Pre-moderated User-generated Content on Public Displays. In Proceedings of the SIGCHI Conference on Human Factors in Computing
Systems (CHI '14). ACM, New York, NY, USA, 1435-1438. DOI :

http://dx.doi.org/10.1145/2556288.2557186

12. Morgan Harvey, Marc Langheinrich, and Geoff Ward. 2016. Remembering through lifelogging: A survey of human memory augmentation. Pervasive and Mobile Computing 27 (2016), 14 -26. DOI :

http://dx.doi.org/https:

//doi.org/10.1016/j.pmcj.2015.12.002

13. Elaine M. Huang, Anna Koster, and Jan Borchers. 2008. Overcoming Assumptions and Uncovering Practices: When Does the Public Really Look at Public Displays?. In Pervasive Computing, Jadwiga Indulska, Donald J. Patterson, Tom Rodden, and Max Ott (Eds.). Springer Berlin Heidelberg, Berlin, Heidelberg, 228-243.

14. Adrian Kirkwood and Linda Price. 2014. Technology-enhanced learning and teaching in higher education: what is "enhanced" and how do we know? A critical literature review. Learning, Media and Technology 39, 1 (2014), 6-36. DOI : http://dx.doi.org/10.1080/17439884.2013.770404

15. Nemanja Memarovic, Marc Langheinrich, Elisa Rubegni, Andreia David, and Ivan Elhart. 2012. Designing interacting places for a student community using a communicative ecology approach. In Proceedings of the 11th International Conference on Mobile and Ubiquitous Multimedia. ACM, New York, NY, USA, 43.

16. T. Ojala, V. Kostakos, H. Kukka, T. Heikkinen, T. Linden, M. Jurmu, S. Hosio, F. Kruger, and D. Zanni. 2012. Multipurpose Interactive Public Displays in the Wild: Three Years Later. Computer 45, 5 (May 2012), 42-49. DOI : http://dx.doi.org/10.1109/MC.2012.115

17. Timo Ojala, Hannu Kukka, Tomas Lindén, Tommi Heikkinen, Marko Jurmu, Simo Hosio, and Fabio Kruger. 2010. UBI-Hotspot 1.0: Large-Scale Long-Term Deployment of Interactive Public Displays in a City Center. In Proceedings of the 2010 Fifth International Conference on Internet and Web Applications and Services (ICIW' 10). IEEE Computer Society, Washington, DC, USA, 285-294. DOI: http://dx.doi.org/10.1109/ICIW. 2010.49

18. Philipp Panhey, Tanja Döring, Stefan Schneegass, Dirk Wenig, and Florian Alt. 2015. What People Really Remember: Understanding Cognitive Effects When Interacting with Large Displays. In Proceedings of the 2015 International Conference on Interactive Tabletops \& Surfaces (ITS '15). ACM, New York, NY, USA, 103-106. DOI: http://dx.doi.org/10.1145/2817721.2817732

19. Peter Peltonen, Esko Kurvinen, Antti Salovaara, Giulio Jacucci, Tommi Ilmonen, John Evans, Antti Oulasvirta, and Petri Saarikko. 2008. It's Mine, Don'T Touch!: Interactions at a Large Multi-touch Display in a City Centre. In Proceedings of the SIGCHI Conference on Human Factors in Computing Systems (CHI '08). ACM, New York, NY, USA, 1285-1294. DOI: http://dx.doi.org/10.1145/1357054.1357255 
20. Abigail J. Sellen and Steve Whittaker. 2010. Beyond Total Capture: A Constructive Critique of Lifelogging. Commun. ACM 53, 5 (May 2010), 70-77. DOI : http://dx.doi.org/10.1145/1735223.1735243

21. O. Storz, A. Friday, N. Davies, J. Finney, C. Sas, and J. Sheridan. 2006. Public Ubiquitous Computing Systems:
Lessons from the e-Campus Display Deployments. IEEE Pervasive Computing 5, 3 (July 2006), 40-47. DOI:

http://dx.doi.org/10.1109/MPRV.2006.56

22. Stuart Webb. 2007. The effects of repetition on vocabulary knowledge. Applied linguistics 28, 1 (2007), 46-65. 